\title{
Rectal separation using hydroxypropyl methylcellulose in intracavitary brachytherapy of cervical cancer: an innovative approach
}

\author{
Swapnendu Basu, MD, DNB', Kazi Sazzad Manir, MD, DNB², Abhishek Basu, MD, DNB³, Koushik Ghosh, Msc, Dip. R.P.3 \\ 'Department of Radiotherapy, Medical Cancer Hospital, Siliguri, Darjeeling, ${ }^{2}$ R.M. O. cum Clinical Tutor, Department of Radiotherapy. \\ R.G. Kar Medical College \& Hospitals, Kolkata, ${ }^{3}$ Department of Radiotherapy, R.G. Kar Medical College \& Hospitals, Kolkata, India
}

\begin{abstract}
Purpose: This study was initiated to prove feasibility of hydrogel application in recto-vaginal space in intracavitary brachytherapy (ICBT) of cervical cancer in order to reduce rectal toxicity.

Material and methods: In a case of stage IIB cervical cancer, after external beam radiotherapy (EBRT), we planned ICBT 7 Gy $\times 3$ fractions. In $1^{\text {st }}$ fraction (Plan 1), due to narrow separation between rectum and cervix $(0.18 \mathrm{~cm})$, only 5 Gy was delivered at point A (with high-risk clinical target volume [HR-CTV] $\mathrm{D}_{90} 5.94 \mathrm{~Gy}$, intermediate risk clinical target volume [IR-CTV] $\mathrm{D}_{90} 4.54 \mathrm{~Gy}$, rectum $\mathrm{D}_{2 \mathrm{cc}} 5.72 \mathrm{~Gy}$, bladder $\mathrm{D}_{2 \mathrm{cc}} 5.52 \mathrm{~Gy}$, and sigmoid colon $\left.5.82 \mathrm{~Gy}\right)$. In $2^{\text {nd }}$ fraction (Plan 2), interstitial brachytherapy (ISBT) was attempted. For the prescription of 5 Gy, we get dose levels almost similar to the $1^{\text {st }}$ insertion: HR-CTV $\mathrm{D}_{90}(6.7 \mathrm{~Gy})$, IR-CTV $\mathrm{D}_{90}(3.06 \mathrm{~Gy})$, bladder $\mathrm{D}_{2 \mathrm{cc}}(5.7 \mathrm{~Gy})$, rectum $\mathrm{D}_{2 \mathrm{cc}}(4.8 \mathrm{~Gy})$, sigmoid colon $\mathrm{D}_{2 \mathrm{cc}}(1.3 \mathrm{~Gy})$ (separation $=0.23 \mathrm{~cm}$ ). During $3^{\text {rd }}$ fraction (Plan 3), prior doing interstitial insertion, we instilled $50 \mathrm{cc}$ of hydroxypropyl methylcellulose $\left(\right.$ Viscomet $\left.^{\circledR}\right)$ up to the tip of recto-vaginal septum. A repeat computed tomography (CT) scan was done 4 hours after Plan 3 treatment and it was re-planned (Plan 4) to find out migration of hydrogel if any and its dosimetric impact.

Results: 9 Gy was delivered to point A with a separation of $1.1 \mathrm{~cm}$ in Plan 3 (with HR-CTV D 90 16.4 Gy, IR-CTV $\mathrm{D}_{90} 11.3 \mathrm{~Gy}$, rectum $\mathrm{D}_{2 \mathrm{cc}} 3.6 \mathrm{~Gy}$, bladder $\mathrm{D}_{2 \mathrm{cc}} 6.9 \mathrm{~Gy}$, and sigmoid colon $2.2 \mathrm{~Gy}$ ). We achieved an optimum cumulative $\mathrm{EQD}_{2}$ dose (HR-CTV D 90.4 Gy, IR-CTV D $90.1 \mathrm{~Gy}$, rectum $\mathrm{D}_{2 \mathrm{cc}} 67.7 \mathrm{~Gy}$, bladder $\mathrm{D}_{2 \mathrm{cc}} 73.2 \mathrm{~Gy}$, and sigmoid colon 59.3 Gy). Hydrogel volume was decreased in Plan 4 without a major dosimetric changes.

Conclusions: Hydrogel instillation is a useful tool for recto-vaginal separation during cervical cancer brachytherapy. It increases therapeutic ratio without any adverse event.

J Contemp Brachytherapy 2016; 8, 5: 399-403 DOI: 10.5114/jcb.2016.62951
\end{abstract}

Key words: brachytherapy, cervical cancer, rectal toxicity.

\section{Purpose}

Intracavitary brachytherapy (ICBT) is important part of definitive radiotherapy protocol of cervical cancer patients. Late adverse events of rectum and bladder are issues of major concern in ICBT $[1,2,3,4,5,6,7]$. Feasibility to conform dose to the target in image based brachytherapy allowed clinicians to adapt dose distribution in organs at risk and tumor in each fraction. Centers practicing image based brachytherapy showed improved dose distribution and outcome, and decreased morbidity. In cervical cancer patients, the cumulative overall and $\geq 2$ grade rectal toxicity has been recorded in $12-19 \%$ of patients $[5,6,7,8,9]$.

From basic radiation protection principles, it is wel known that increasing the distance is a simple and effective way to reduce radiation exposure. The interest in recent years to physically separate the rectum from the prostate and thereby reduce the rectal radiation dose has been significant. Injection of human collagen into the space between prostate and rectum in prostate cancer patients have shown decline of rectal dose by $25-50 \%$ $[10,11]$. Till date, there is only a single published case report of this new technique for patients with cervical cancer undergoing external beam radiotherapy (EBRT). Nevertheless, Marnitz et al. in their study did not discuss the dosimetric impact of this intervention [12]. The present study was initiated to prove the feasibility of hydrogel application in ICBT of cervical cancer in order to reduce rectal toxicity. To the best of our knowledge, the present study is the first report of rectal hydrogel application in ICBT of cervical cancer.

Address for correspondence: Kazi Sazad Manir, MD, DNB, Department of Radiotherapy, R.G.Kar Medical Received: 17.01 .2016 College and Hospitals, 1 Khudiram Bose Sarani, Kolkata 70004, India, phone: +91-9432187498, 


\section{Material and methods}

A 56 years old multiparous woman was presented with cervical cancer FIGO (International Federation of Gynecology and Obstetrics) stage IIB. After an magnetic resonance imaging (MRI) confirmed her staging, she was

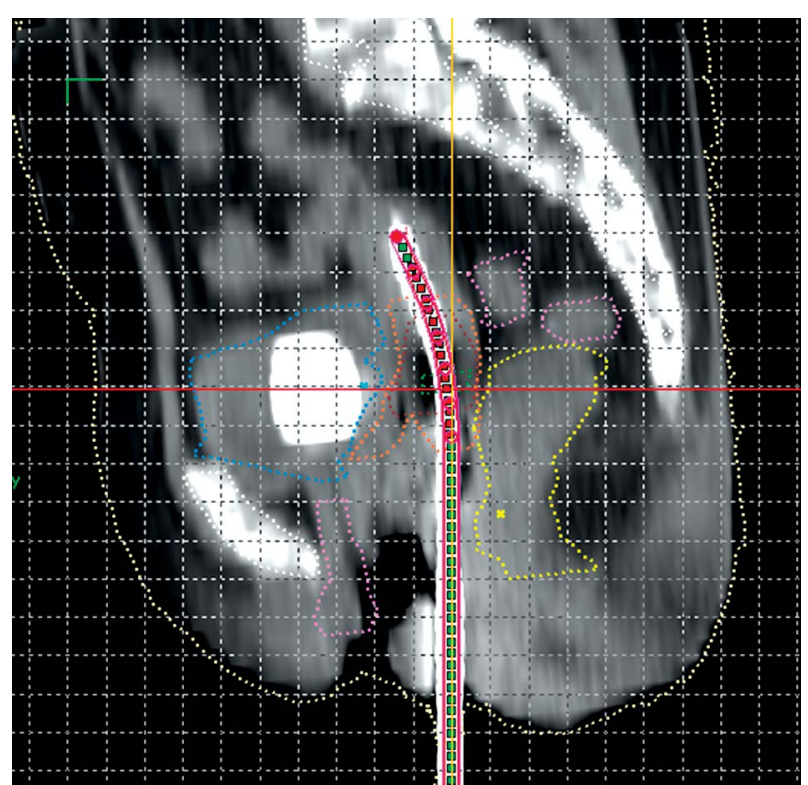

Fig. 1. Sagittal view of computed tomography scan of Plan 1 showing interrelationship of contoured volumes e.g. highrisk clinical target volume (HR-CTV) (brown), intermediate risk clinical target volume (IR-CTV) (orange), rectum (yellow), sigmoid colon (pink), bladder (blue), and urethra (violet)

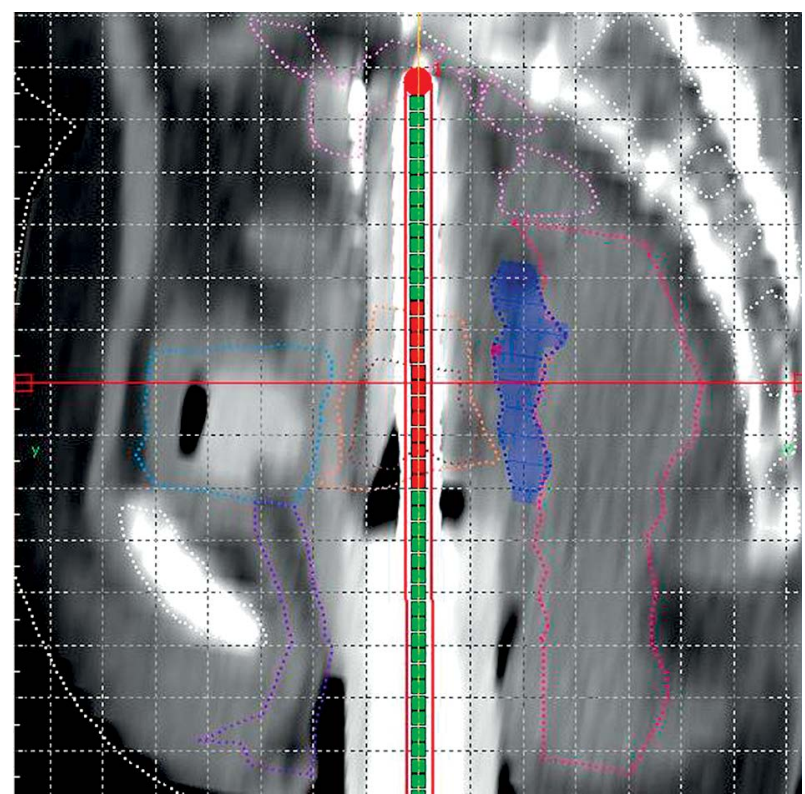

Fig. 2. Sagittal view of computed tomography scan of Plan 3 showing interrelationship of contoured volumes e.g. highrisk clinical target volume (HR-CTV) (brown), intermediate risk clinical target volume (IR-CTV) (orange), rectum (deep pink), sigmoid colon (light pink), bladder (light blue), urethra (violet), and Viscomet ${ }^{\circledR}$ (deep blue) treated with EBRT to a dose of 50.4 Gy in 28 fractions (1.8 Gy/fraction) over 5 weeks along with weekly concurrent injections of cisplatin $40 \mathrm{mg} / \mathrm{m}^{2}$. Post EBRT gynecological and imaging assessment showed no residual tumor. She was therefore planned for ICBT. Initially, we planned to deliver a dose of $7 \mathrm{~Gy} \times 3$ fractions, one fraction per week. According to our institutional protocol, the patient was admitted one day prior to ICBT application for bowel preparation (digestive enzymes, bisacodyl tablets, and enema). Intracavitary brachytherapy application was done under spinal anesthesia with standard FletcherSuit-Delclos (FSD) applicators on 03/09/2014. Computed tomography (CT) of pelvis was done followed by contouring of organs at risk (OAR) (i.e. urinary bladder, rectum, and sigmoid colon), gross tumor volume at brachytherapy (GTVB), high risk clinical target volume (HR-CTV), and intermediate risk clinical target volume (IR-CTV) $[13,14,15]$ using the high-dose-rate (HDR) plus v2.6 treatment planning system (Eckert \& Zieglar, Bebig GmBH, Germany). Due to close proximity of the rectum with the target volume, in spite of optimization, we were able to prescribe only 5 Gy to point $A$ (with doses to HR-CTV $D_{90}$ $5.94 \mathrm{~Gy}$, IR-CTV $\mathrm{D}_{90} 4.54 \mathrm{~Gy}$, rectum $\mathrm{D}_{2 \mathrm{cc}} 5.72 \mathrm{~Gy}$, bladder $\mathrm{D}_{2 \mathrm{cc}} 5.52 \mathrm{~Gy}$, and sigmoid colon $5.82 \mathrm{~Gy}$; Plan 1). On reviewing the insertion, internal anatomy, and planning, we found out that the key problem for the high rectal and sigmoid doses was the wall separation between the rec-

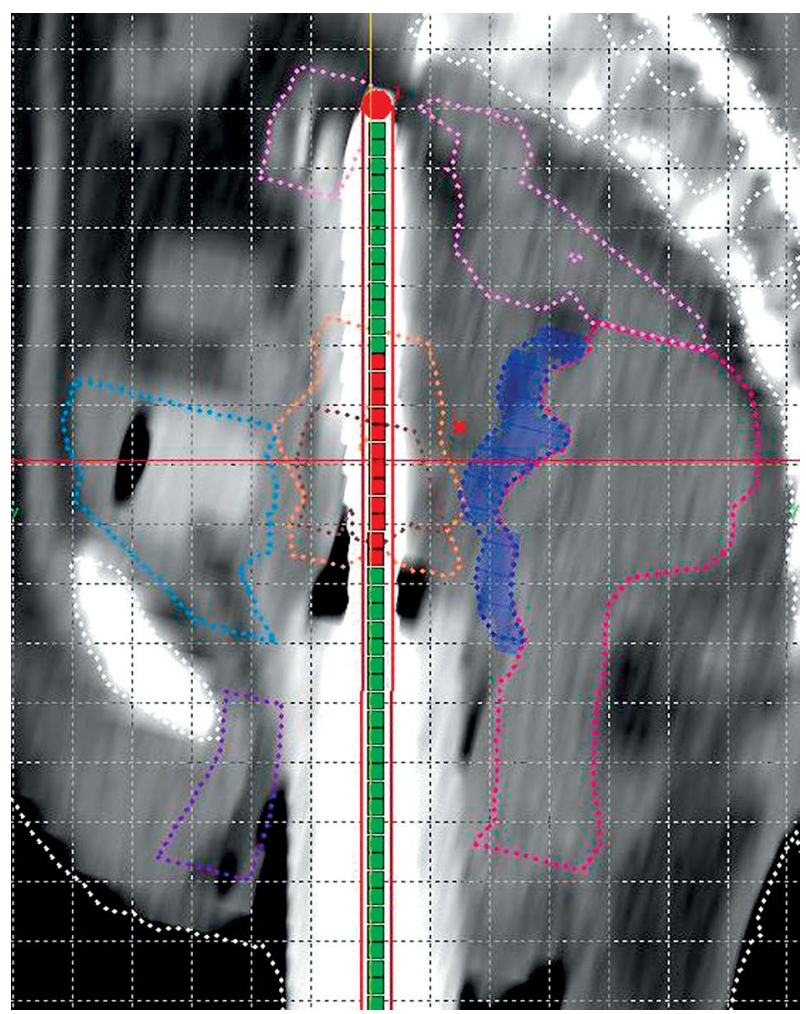

Fig. 3. Sagittal view of computed tomography scan of Plan 4 showing interrelationship of contoured volumes e.g. highrisk clinical target volume (HR-CTV) (brown), intermediate risk clinical target volume (IR-CTV) (orange), rectum (deep pink), sigmoid colon (light pink), bladder (light blue), urethra (violet), and Viscomet ${ }^{\circledR}$ (deep blue) 
tum and the cervix, which was very narrow (average $=$ $0.18 \mathrm{~cm}$ ) (Figure 1). Treatment was delivered through ${ }^{60} \mathrm{Co}$ HDR brachytherapy unit (Multisource, Eckert \& Zieglar, Bebig GmBH, Germany).

In the subsequent fraction, with informed consent of the patient, we decided to go for interstitial brachytherapy (ISBT) implant. We inserted 10 metallic needles in two circles and the FSD tandem (previously used angulation) through Syed Neblett gynecological interstitial template on 09/09/2014. However, we failed to deliver the desired 7 Gy and prescribed only 5 Gy instead. For the prescription of $5 \mathrm{~Gy}$, the dose levels were almost similar to the $1^{\text {st }}$ insertion: HR-CTV $\mathrm{D}_{90}(6.7 \mathrm{~Gy}), \mathrm{IR}-\mathrm{CTV} \mathrm{D}_{90}(3.06 \mathrm{~Gy})$ bladder $\mathrm{D}_{2 \mathrm{cc}}(5.7 \mathrm{~Gy})$, rectum $\mathrm{D}_{2 \mathrm{cc}}(4.8 \mathrm{~Gy})$, and sigmoid colon $\mathrm{D}_{2 \mathrm{cc}}(1.3 \mathrm{~Gy})$. We could not deliver 7 Gy prescription due to high doses to the OAR in order to keep the
$\mathrm{EQD}_{2}$ (equivalent dose in $2 \mathrm{~Gy} /$ fraction adding EBRT and brachytherapy doses, and considering $\alpha / \beta$ for OAR $=3$ Gy and for tumor $=10 \mathrm{~Gy}$ ) of bladder $\mathrm{D}_{2 \mathrm{cc}}<90 \mathrm{~Gy}, \mathrm{EQD}_{2}$ rectum $\mathrm{D}_{2 \mathrm{cc}}$ and sigmoid colon $\mathrm{D}_{2 \mathrm{cc}}<70 \mathrm{~Gy}$ as mandated in our institutional protocol. Moreover, in order to get HR-CTV EQD ${ }_{2}$ of $D_{90}>85 \mathrm{~Gy}$, which is our institutional protocol, this prescription (if followed also in third fraction) would be suboptimal (Plan 2). On reviewing all the steps of the second fraction, we observed that the problem remained the same like the first fraction i.e. narrow space (average $=0.23 \mathrm{~cm}$ ) between the rectum and the cervix

Therefore, for the third fraction, with informed consent, we decided to instill hydrogel in the recto-vaginal space. On 12/09/2014 under spinal anesthesia, the patient underwent ISBT with Syed Neblett template (6 needles in inner circle of obturator and central FSD tandem).

Table 1. Fraction wise and total dose distribution of all insertions

\begin{tabular}{|c|c|c|c|c|c|c|}
\hline Insertions & $\begin{array}{l}1^{\text {st }} \text { insertion } \\
\text { Plan } 1\end{array}$ & $\begin{array}{l}2^{\text {nd }} \text { insertion } \\
\text { Plan } 2\end{array}$ & $\begin{array}{l}3^{\text {rd }} \text { insertion } \\
\text { Plan } 3\end{array}$ & $\begin{array}{c}\text { Absolute } \\
\text { difference } \\
\text { from Plan } 3 \text { - } \\
\text { Plan } 2 \text { dose }\end{array}$ & $\begin{array}{c}\text { Relative } \\
\text { difference } \\
\text { from Plan } 3- \\
\text { Plan } 2 \text { dose }\end{array}$ & $\begin{array}{c}\mathrm{EQD}_{2} \\
(\mathrm{EBRT}+\text { all } \mathrm{BT})\end{array}$ \\
\hline Prescription dose & 5 Gy & $5 \mathrm{~Gy}$ & 9 Gy & & $\%$ & \\
\hline \multicolumn{7}{|l|}{ All doses in Gy } \\
\hline \multicolumn{7}{|l|}{$\operatorname{HR}-\operatorname{CTV}(\alpha / \beta=10)$} \\
\hline$D_{90}$ & 5.9 & 6.7 & 16.4 & 9.7 & 48.2 & 98.4 \\
\hline$D_{100}$ & 3.8 & 4.5 & 10.2 & 5.7 & 23.3 & 72.1 \\
\hline$V_{100}(\%)$ & 96.3 & 99.3 & 100 & 0.7 & & 98.5 \\
\hline \multicolumn{7}{|l|}{$\operatorname{IRCTV}(\alpha / \beta=10)$} \\
\hline $\mathrm{D}_{90}$ & 4.5 & 4.5 & 11.3 & 6.8 & 35.6 & 76.1 \\
\hline$D_{100}$ & 2.7 & 3.1 & 4.1 & 1.0 & -16.4 & 56.2 \\
\hline$V_{100}(\%)$ & 85.5 & 83.1 & 95.7 & 12.6 & & 88.1 \\
\hline \multicolumn{7}{|l|}{ Bladder $(\alpha / \beta=3)$} \\
\hline $\mathrm{D}_{0.1 c c}$ & 8.4 & 5.7 & 11.2 & 5.5 & 10.4 & 106.3 \\
\hline $\mathrm{D}_{1 \mathrm{cc}}$ & 6.2 & 4.1 & 8 & 3.9 & 6.9 & 80.2 \\
\hline$D_{2 c c}$ & 5.5 & 3.6 & 6.9 & 3.3 & 4.7 & 73.2 \\
\hline \multicolumn{7}{|l|}{$\operatorname{Rectum}(\alpha / \beta=3)$} \\
\hline $\mathrm{D}_{0.1 \mathrm{cc}}$ & 9.6 & 11.0 & 4.9 & -6.1 & -156.6 & 108.1 \\
\hline $\mathrm{D}_{1 \mathrm{cc}}$ & 6.8 & 6.2 & 4 & -2.2 & -79.6 & 75.7 \\
\hline$D_{2 c c}$ & 5.7 & 4.8 & 3.6 & -1.2 & -56 & 67.7 \\
\hline \multicolumn{7}{|c|}{ Sigmoid colon $(\alpha / \beta=3)$} \\
\hline $\mathrm{D}_{0.1 \mathrm{cc}}$ & 9.6 & 1.8 & 3.1 & 1.3 & -1.6 & 75.1 \\
\hline $\mathrm{D}_{1 \mathrm{cc}}$ & 6.9 & 1.4 & 2.4 & 1.3 & -1.3 & 62.9 \\
\hline $\mathrm{D}_{2 \mathrm{cc}}$ & 5.9 & 1.3 & 2.2 & 1.1 & -1.6 & 59.3 \\
\hline
\end{tabular}

$E B R T$ - external beam radiotherapy, $B T$ - brachytherapy, $E Q D_{2}$ - equivalent dose in 2 Grey per fraction, HR-CTV - high risk clinical target volume, $D_{90}$ - minimum dose received by $90 \%$ of the volume expressed as a percentage of the prescription dose, $D_{100}$ - minimum dose received by $100 \%$ of the volume expressed as a percentage of the prescription dose, $V_{100}$-percentage of the target volume covered by $100 \%$ of the prescription dose, IR-CTV-intermediate risk clinical target volume, $D_{0.1 c c}$ - the minimum doses to the highest irradiated $0.1 \mathrm{cc}$ volume, $D_{1 \mathrm{cc}}$ - the minimum doses to the highest irradiated $1 \mathrm{cc}$ volume, $D_{2 \mathrm{cc}}-$ the minimum doses to the highest irradiated 2 cc volume 
During this procedure, instillation of the hydrogel was also performed. After Foleys catheterization, a Cusco's self-retaining bivalve speculum was introduced, the os was identified, and a $50 \mathrm{cc}$ syringe was filled with hydroxypropyl methylcellulose (Viscomet $\left.{ }^{\circledR}\right)$. The needle of the syringe was inserted through the recto-vaginal septum. One index finger was kept per rectally to feel the passage of the needle of instilling syringe. Slowly, the hydrogel was instilled along the recto-vaginal septum. The needle

Table 2. Comparative dosimetry of Plan 3 and Plan 4

\begin{tabular}{|c|c|c|c|}
\hline Insertions & $\begin{array}{c}3^{\text {rd }} \text { insertion } \\
\text { Plan } 3\end{array}$ & Plan 4 & Difference \\
\hline Prescription dose & 9 Gy & 9 Gy & \\
\hline \multicolumn{4}{|l|}{$\operatorname{HR}-\operatorname{CTV}(\alpha / \beta=10)$} \\
\hline $\mathrm{D}_{90}$ & 16.4 & 15.63 & -0.77 \\
\hline$D_{100}$ & 10.2 & 10.36 & 0.15 \\
\hline $\mathrm{V}_{100}(\%)$ & 100 & 100 & 0 \\
\hline \multicolumn{4}{|l|}{$\operatorname{IRCTV}(\alpha / \beta=10)$} \\
\hline $\mathrm{D}_{90}$ & 11.3 & 9.12 & -2.18 \\
\hline $\mathrm{D}_{100}$ & 4.1 & 4.58 & 0.48 \\
\hline $\mathrm{V}_{100}(\%)$ & 95.7 & 90.6 & -5.1 \\
\hline \multicolumn{4}{|l|}{ Bladder $(\alpha / \beta=3)$} \\
\hline $\mathrm{D}_{0.1 c c}$ & 11.2 & 10.63 & -0.57 \\
\hline $\mathrm{D}_{1 \mathrm{Cc}}$ & 8 & 8.17 & 0.17 \\
\hline$D_{2 c c}$ & 6.9 & 7.06 & 0.66 \\
\hline \multicolumn{4}{|l|}{$\operatorname{Rectum}(\alpha / \beta=3)$} \\
\hline $\mathrm{D}_{0.1 \mathrm{ccc}}$ & 4.9 & 5.16 & 0.26 \\
\hline $\mathrm{D}_{1 \mathrm{cc}}$ & 4 & 4.19 & 0.19 \\
\hline $\mathrm{D}_{2 c c}$ & 3.6 & 3.71 & 0.11 \\
\hline \multicolumn{4}{|c|}{ Sigmoid colon $(\alpha / \beta=3)$} \\
\hline $\mathrm{D}_{0.1 c c}$ & 3.1 & 1.97 & -1.13 \\
\hline $\mathrm{D}_{1 \mathrm{Cc}}$ & 2.4 & 1.63 & -0.73 \\
\hline$\overline{D_{2 c c}}$ & 2.2 & 1.47 & -0.73 \\
\hline $\begin{array}{l}\text { Hydrogel contour } \\
\text { volume (cc) }\end{array}$ & 9.3 & 7.2 & -2.1 \\
\hline
\end{tabular}

Hydrogel volume dimension:

supero-inferior $x \quad 4 \times 1 \times 2.5 \quad 5 \times 0.8 \times 2$

antero-posterior $x$

medio-lateral $(\mathrm{cm})$

$H R$-CTV - high risk clinical target volume, $D_{90}$ - minimum dose received by $90 \%$ of the volume expressed as a percentage of the prescription dose, $D_{100}-$ minimum dose received by $100 \%$ of the volume expressed as a percentage of the prescription dose, $V_{100}$ - percentage of the target volume covered by $100 \%$ of the prescription dose, IR-CTV - intermediate risk clinical target volume, $D_{01 c c}$ the minimum doses to the highest irradiated $0.1 \mathrm{cc}$ volume, $D_{1 \mathrm{cc}}$ - the minimum doses to the highest irradiated $1 \mathrm{cc}$ volume, $D_{2 c c}$ - the minimum doses to the highest irradiated 2 cc volume was then carefully withdrawn while pushing the hydrogel as high as possible into the septum (Figure 2). After this, the ISBT insertion was done. The patient underwent a plain CT scan of pelvis with $2.5 \mathrm{~mm}$ slice thickness. The whole procedure was uneventful. We contoured the hydrogel area separately (Plan 3 ). We did a repeat CT scan with applicator in situ after the treatment was over (about 4 hours after hydrogel insertion) to find out any migration of hydrogel. We also contoured and planned virtually on this image dataset (Plan 4) and compared it dosimetrically with the third fraction insertion (Figure 3).

\section{Results}

The instilled hydrogel area was optimally visible on planning CT between the anterior rectal wall and the posterior vaginal wall. The average width of separation was $1.1 \mathrm{~cm}$, length of $5 \mathrm{~cm}$. The patient was planned and we were able to deliver 9 Gy with an optimized dose distribution in the HR-CTV and OAR. Fraction-wise and total doses of CTV and OAR including the relative and absolute dosimetric changes from Plan 2 \& Plan 3 are detailed in Table 1. Dosimetric comparison of Plan 3 and Plan 4 are given in Table 2 . The hydrogel volume was decreased on Plan 4 with superior-inferior expansion $(9.3 \mathrm{cc}$ in Plan 3 [supero-inferior $\times$ anterio-posterior $\times$ medio-lateral: being $4 \times 1 \times 2.5 \mathrm{~cm}$, respectively] versus $7.2 \mathrm{cc}$ in Plan 4 [ $5 \times 0.8 \times 2 \mathrm{~cm}$ respectively]) without any major change in other dosimetric parameters.

Post implant days were uneventful. The patient was examined after 6 weeks. There was neither a residual tumor nor any adverse event. The patient remains in follow-up till date without any late toxicity and is in complete remission.

\section{Discussion}

Most of the studies using hydrogel for rectal separation are in prostate cancer. In the first published report of this kind, Susil et al. showed that a decrease of rectal $\mathrm{V}_{70}$ Gy significantly from $23 \%$ baseline value to $15 \%, 4 \%$, and $0 \%$ was possible with $5 \mathrm{~mm}, 10 \mathrm{~mm}$, and $15 \mathrm{~mm}$ separation, respectively [10]. This finding was confirmed in further clinical studies $[11,12,13,14,15,16]$. In their study, Prada et al. demonstrated that the measured rectal dose for HDR boost was significantly lower by about 2 Gy in prostate cancer patients [16]. Marnitz et al. demonstrated the use of recto-vaginal separation using hydrogel in EBRT for cervical cancer [12]. They achieved a median separation width of $10 \mathrm{~mm}$, length of $32 \mathrm{~mm}$.

To the best of our knowledge, our study is the first to report about rectal separation using hydrogel in brachytherapy for cervical cancer. We tried to increase the separation between the rectum and the cervical tissue in this difficult case where the septum width was very narrow $(<1 \mathrm{~cm}$ : $0.23 \mathrm{~cm}$ ). The idea was to deliver optimum dose to tumor target, keeping the rectal dose as low as possible. The average separation width achieved was $11 \mathrm{~mm}$. The favorable dosimetric impact is depicted in Table 1 . We observed no adverse events during and after the procedure. The hydrogel volume was easily visible in CT scans with a slight mi- 
gration, and the minor reduction noted in Plan 4 had no major impact on the dosimetric parameters.

\section{Conclusions}

Hydrogel instillation may be a useful tool for rectovaginal separation during brachytherapy of cervical cancer. It appears to increase the therapeutic ratio without any adverse event. Considering this to be the first report of its kind, we plan to prospectively try this approach in selected patients in the future.

\section{Disclosure}

Authors report no conflict of interest.

\section{References}

1. Barillot I, Horiot JC, Maingon P et al. Impact on treatment outcome and late effects of customized treatment planning in cervix carcinomas: baseline results to compare new strategies. Int J Radiat Oncol Biol Phys 2000; 48: 189-200.

2. Chen SW, Liang JA, Yang SN et al. The prediction of late rectal complications following the treatment of uterine cervical cancer by high-dose-rate brachytherapy. Int J Radiat Oncol Biol Phys 2000; 47: 955-961.

3. Eifel PJ, Thoms WW Jr, Smith TL et al. The relationship between brachytherapy dose and outcome in patients with bulky endocervical tumors treated with radiation alone. Int J Radiat Oncol Biol Phys 1994; 28: 113-118.

4. Eifel PJ, Levenback C, Wharton T et al. Time course and incidence of late complications in patients treated with radiation therapy for FIGO stage Ib carcinoma of the uterine cervix. Int J Radiat Oncol Biol Phys 1995; 32: 1289-1300.

5. Perez CA, Grigsby PW, Lockett MA et al. Radiation therapy morbidity in carcinoma of the uterine cervix: dosimetric and clinical correlation. Int J Radiat Oncol Biol Phys 1999; 44: 855-866.

6. Sakata K, Nagakura H, Oouchi A et al. High-dose-rate intracavitary brachytherapy: results of analyses of late rectal complications. Int J Radiat Oncol Biol Phys 2002; 54: 1369-1376.

7. Sivaspour Z, Aghamiri MR, Jaberi R et al. Optimum organ volume ranges for organs at risk dose in cervical cancer intracavitary brachytherapy. J Contemp Brachytherapy 2016; 8: 135-142.

8. Georg P, Kirisits C, Goldner G et al. Correlation of dose-volume parameters, endoscopic and clinical rectal side effects in cancer cervix patients treated with definitive radiotherapy including MRI based brachytherapy. Radiother Oncol 2009; 91: 173-180.

9. Chen SW, Liang JA, Hung YC et al. Late toxicities in concurrent chemoradiotherapy using high-dose-rate intracavitary brachytherapy plus weekly cisplatin for locally advanced cervical cancer: a historical cohort comparison against two previous different treatment schemes. Eur J Gynaecol Oncol 2010; 31: 504-509.

10. Susil RC, McNutt TR, DeWeese TL et al. Effects of prostate-rectum separation on rectal dose from external beam radiotherapy. Int J Radiat Oncol Biol Phys 2010; 76: 1251-1258.

11. Nguyen PL, Devlin PM, Beard CJ et al. High-dose-rate brachytherapy for prostate cancer in a previously radiated patient with polyethylene glycol hydrogel spacing to reduce rectal dose: case report and review of the literature. Brachytherapy 2013; 12: 77-83.

12. Marnitz S, Budach V, Weisser F et al. Rectum separation in patients with cervical cancer for treatment planning in primary chemo-radiation. Radiat Oncol 2012; 7: 109.
13. Haie-Meder C, Pötter R, Van Limbergen E et al. Gynaecological (GYN) GEC-ESTRO Working Group (I): Recommendations from Gynaecological (GYN) GEC ESTRO Working Group (I): concepts and terms in 3D image based 3D treatment planning in cervix cancer brachytherapy with emphasis on MRI assessment of GTV and CTV. Radiother Oncol 2005; 74: 235-245.

14. Pötter R, Haie-Meder C, Van Limbergen E et al. GEC ESTRO Working Group. Recommendations from gynaecological (GYN) GEC ESTRO working group (II): Concepts and terms in 3D image based treatment planning in cervix cancer brachytherapy $-3 \mathrm{D}$ dose volume parameters and aspects of 3D image-based anatomy, radiation physics, radiobiology. Radiother Oncol 2006; 78: 67-77.

15. Viswanathan AN, Dimopoulos J, Kirisits C et al. Computed tomography versus magnetic resonance imaging-based contouring in cervical cancer brachytherapy: results of a prospective trial and preliminary guidelines for standardized contours. Int J Radiat Oncol Biol Phys 2007; 68: 491-498.

16. Prada PJ, Fernández J, Martinez AA et al. Transperineal injection of hyaluronic acid in anterior perirectal fat to decrease rectal toxicity from radiation delivered with intensity modulated brachytherapy or EBRT for prostate cancer patients. Int J Radiat Oncol Biol Phys 2007; 69: 95-102. 